\title{
Electroweak phase transition in the scale invariant standard model
}

\author{
Parsa Hossein Ghorbani* \\ Institute for Research in Fundamental Sciences (IPM), School of Particles and Accelerators, \\ P.O. Box 19395-5531, Tehran, Iran
}

(Received 14 June 2018; published 10 December 2018)

\begin{abstract}
In an extension to the scale invariant standard model by two real singlet scalars $s$ and $s^{\prime}$ in addition to the Higgs field, we investigate the strong first-order electroweak phase transition as a requirement for baryogenesis. This is the minimal extension to the scale invariant standard model with two extra degrees of freedom that possesses the physical Higgs mass of $125 \mathrm{GeV}$. The scalar $s^{\prime}$ being stable because of the $\mathbb{Z}_{2}$ discrete symmetry is taken as the dark matter candidate. We then show that the electroweak phase transition is strongly first order, the dark matter relic density takes the desired value $\Omega_{\mathrm{DM}} h^{2} \sim 0.11$, and the constraints from direct detection experiments are respected only if $m_{s^{\prime}} \equiv m_{\mathrm{DM}} \gtrsim 4.5 \mathrm{TeV}$. The model also puts a lower bound on the scalon mass, $m_{s} \gtrsim 200 \mathrm{GeV}$.
\end{abstract}

DOI: 10.1103/PhysRevD.98.115016

\section{INTRODUCTION}

After the discovery of the Higgs particle in July 2012 at the LHC [1,2], the last missing piece of the standard model (SM) prediction, made almost a half-century ago [3,4], was completed. The SM has been tested by the most stringent scrutinies over many different experiments and it has passed them successfully. However, there are a number of issues, either theoretical or experimental/observational, that are not compatible with the SM predictions. The gauge hierarchy problem, the strong first-order electroweak phase transition (EWPT) and other conditions needed in the baryogenesis mechanism, and the problem of dark matter are some examples of unanswered puzzles in the SM. These inconsistencies led people to think of theories beyond the SM such as the GUT, SUSY, etc. Our goal in this paper is to address the above-mentioned SM shortcomings rather in a minimal extension of the scale invariant standard model (MSISM).

The negative Higgs mass term $-m_{H}^{2} H^{\dagger} H$ in the SM potential causes a quadratical divergent term proportional to the energy scale cutoff $\Lambda^{2}$ after including the quantum corrections. In fact, the Higgs mass term is the only term that breaks the classical scale invariance in the SM. Therefore, by omitting the Higgs mass term from the SM potential, we have practically removed the problem of

\footnotetext{
*parsaghorbani@gmail.com
}

Published by the American Physical Society under the terms of the Creative Commons Attribution 4.0 International license. Further distribution of this work must maintain attribution to the author(s) and the published article's title, journal citation, and DOI. Funded by SCOAP. gauge hierarchy. ${ }^{1}$ In the seminal paper of Coleman and Weinberg [7], it was shown that in a scale invariant gauge theory the radiative corrections break the scale invariance and that triggers the spontaneous symmetry breaking. Following their work, Gildener and Weinberg [8] argued that in the SISM the radiative corrections break the electroweak symmetry and thereby the Higgs mechanism is restored for the SISM. The SISM with only one classically massless scalar (Higgs) cannot be realistic because, as computed by Gildener and Weinberg from the quantum corrections, the Higgs mass can be just as light as around $5 \mathrm{GeV}$, which is far lighter than the observed Higgs mass, $m_{H} \sim 125 \mathrm{GeV}$. As discussed in [8], in general among the $n$ scalars, in addition to the Higgs scalar in the extended SISM, there is at least one heavy scalar that may be interpreted as the Higgs particle and there is one classically massless scalar that is dubbed the "scalon." In this paper we add only two extra scalars to the SISM, as the most minimal scale invariant extension of SISM that contains the correct Higgs mass. There are papers in the literature (see, for instance, [9-11]) that have sought a similar scope, but none of them has presented an analytical investigation of the electroweak phase transition and, furthermore, they have usually involved extra fermionic degrees of freedom in the hidden sector. Among papers which address the problem of dark matter using a scale invariant extension of the SM with multiple scalars, one may refer to Ref. [12], in which the detectability of the

\footnotetext{
${ }^{1}$ Even with the vanishing Higgs mass, the quadratically divergent term depends on the regularization scheme and may reappear due to the quantum corrections [5]. Nevertheless, in the dimensional regularization scheme, the Higgs remains massless to all orders of perturbation [6].
} 
real scalar in direct detection experiments is examined. In, for instance, Ref. [13], on the other hand, the strongly firstorder electroweak phase transition is studied in the scale invariant SM with additional isospin singlet scalar fields. In the current work, we are interested only in studying the pure scalar and minimal extension of the SISM by investigating both the dark matter (DM) and the EWPT at the same time.

This paper is organized as follows. In the next section we build up the model extending the SISM with two real scalars. Then in Sec. III we derive the critical temperature and the washout criterion for the electroweak phase transition. In Sec. IV the stability conditions are given and then Sec. V will be on dark matter computations. Finally in Sec. VI we examine the model with the experimental bounds on dark matter elastic scattering off the nucleus. We conclude in Sec. VII.

\section{MINIMAL EXTENSION OF SCALE INVARIANT STANDARD MODEL}

In the SM, if we set the Higgs mass term to zero, the only term remaining in the Higgs potential will be $\lambda\left(H^{\dagger} H\right)^{2}$ or $\lambda h^{4}$ after gauging away three components of the Higgs doublet. In the Gildener-Weinberg notation [8], the scalar potential with $n$ scalars, $s_{i}$, is shown as $1 / 24 \lambda_{i j k l} s_{i} s_{j} s_{k} s_{l}$, where $\lambda_{i j k l}$ denotes the coupling. As discussed in [14], in order to have a scale invariant version of the standard model possessing a Higgs doublet with the observed Higgs mass of $125 \mathrm{GeV}$, as well as other SM particles with their physical masses, at least two more scalars (singlet) must be added to the theory. The reason comes from Eq. (4.6) of [8], where the scalon gaining mass through the radiative corrections depends only on the masses of the Higgs particle, the gauge bosons, and the mass of the top quark. In the absence of any additional scalar except the Higgs and the scalon, this expression will be negative. In this paper, therefore, we stay in the most minimal potential with only two extra scalars, which we call here $s$ and $s^{\prime}$. We also assume that these scalars appear with the $\mathbb{Z}_{2}$ symmetry in the potential to attribute the latter to the dark matter candidate,

$$
\begin{aligned}
V_{\mathrm{tr}}\left(h, s, s^{\prime}\right)= & \frac{1}{4} \lambda_{h} h^{4}+\frac{1}{2} \lambda_{h s} s^{2} h^{2}+\frac{1}{4} \lambda_{s} s^{4}+\frac{1}{2} \lambda_{s s^{\prime}} s^{2} s^{\prime 2} \\
& +\frac{1}{4} \lambda_{s^{\prime}} s^{\prime 4}
\end{aligned}
$$

where $h$ stands for the Higgs field, $H^{\dagger}=\frac{1}{\sqrt{2}}(0 h)$. At high temperature, above the electroweak phase transition temperature, the theory lives in its symmetric phase and the vacuum expectation values (VEVs) of the fields are temperature dependent. Let us assign the VEV of each field as

$$
v_{h}(T) \equiv\langle h\rangle, \quad v_{s}(T) \equiv\langle s\rangle, \quad v_{s^{\prime}}(T) \equiv\left\langle s^{\prime}\right\rangle .
$$

We require that the vacuum expectation values after the phase transition be $\left(v_{h}=246 \mathrm{GeV}, v_{s} \neq 0, v_{s^{\prime}}=0\right)$ so that the scalar field $s^{\prime}$ remains stable because of the $\mathbb{Z}_{2}$ symmetry and thus it can play the role of the dark matter candidate. We see later that the value of $v_{s}$ is not fixed and it depends on the value of the couplings in the model.

In the Lagrangian in Eq. (1), the term $\lambda_{h s^{\prime}} h^{2} s^{2}$ could in principle be considered because $s^{\prime}$ undergoes no VEV. Although this term contributes in the DM relic density, we know from the singlet Higgs portal model (SHP) that $\lambda_{h s^{\prime}}$ must be small to evade the direct detection bounds. It is therefore reasonable to assume that vanishing. The inclusion of the $\mathbb{Z}_{2}$ odd terms $s^{\prime} s^{3}$ and $s^{\prime 3} s$ in the Lagrangian is allowed by the scale invariance but will lead to the decay of the DM scalar $s^{\prime}$ and the observed relic density will not be obtained; hence we avoided such terms in the Lagrangian.

In the scale invariant standard model, the flat direction is defined as the direction along which the tree-level potential is vanishing. This condition is equivalent to imposing the Ward identity of the scale symmetry in a scalar theory [15]. The flat direction for the potential in Eq. (1) is obtained via a rotation in the $(h, s)$ space by the angle $\alpha$,

$$
\cos ^{2} \alpha=-\frac{\lambda_{h s}}{\lambda_{h}-\lambda_{h s}}, \quad \lambda_{h s}^{2}-\lambda_{h} \lambda_{s}=0 .
$$

The mass matrix (being meaningful after the phase transition at low temperature) is off-diagonal only in the $(h, s)$ block. This is because of our special choice; we have taken a nonzero VEV for $h$ and $s$ and vanishing VEV for $s^{\prime}$. Finally, the mass eigenvalues after the EWPT read

$m_{h}^{2}=2 v_{h}^{2}\left(\lambda_{h}-\lambda_{h s}\right), \quad m_{s}^{2}=0, \quad m_{s^{\prime}}^{2}=-v_{h}^{2} \frac{\lambda_{h} \lambda_{s s^{\prime}}}{\lambda_{h s}}$.

The one-loop correction at zero temperature gives a small mass to the classically massless eigenstate $s$, the socalled scalon field [8]:

$\delta m_{s}^{2}=\frac{-\lambda_{h s}}{32 \pi^{2} m_{h}^{2}}\left(m_{h}^{4}+m_{s^{\prime}}^{4}+6 m_{W}^{4}+3 m_{Z}^{4}-12 m_{t}^{4}\right)$.

Without the introduction of the second singlet scalar $s^{\prime}$ while having the observed Higgs mass of $125 \mathrm{GeV}$ and the correct masses for the top quark and gauge bosons, the mass correction to the scalon field $s$ could not be positive. Now by means of the radiative correction, the scalon can be interpreted as the mediator in DM models. This can be seen from Eq. (5). For more details, see [14].

The one-loop effective potential consists of the tree-level potential in Eq. (1), the Coleman-Weinberg one-loop correction at zero temperature, and the one-loop correction at finite temperature, 


$$
V_{\text {eff }}=V_{\text {tr }}+V_{0}^{1 \text {-loop }}+V_{T}^{1 \text {-loop }} .
$$

If $T \gg m_{i}$ with $m_{i}$ the tree-level mass of particle $i$, the oneloop thermal contribution is approximated as $V_{T}^{1 \text {-loop }} \simeq C T^{2} \phi^{2}$ and the total one-loop effective potential becomes [16]

$$
V_{\text {eff }} \simeq-\frac{1}{4} B \phi^{4}+\frac{1}{2} B \phi^{4} \log \frac{\phi^{2}}{v_{\phi}^{2}}+C T^{2} \phi^{2},
$$

where $\phi$ is the radial field in the polar coordinate system, i.e., $(h, s) \equiv(\phi \cos \alpha, \phi \sin \alpha)^{2}$; hence $v_{\phi}^{2}=v_{h}^{2}+v_{s}^{2}$. The coefficients $B$ and $C$ are

$$
\begin{gathered}
B=\frac{1}{64 \pi^{2} v_{\phi}^{4}}\left(m_{h}^{4}+m_{s^{\prime}}^{4}+6 m_{W}^{4}+3 m_{Z}^{4}-12 m_{t}^{4}\right) \\
C=\frac{1}{12 v_{\phi}^{2}}\left(m_{h}^{2}+m_{s^{\prime}}^{2}+6 m_{W}^{2}+3 m_{Z}^{2}+6 m_{t}^{2}\right) .
\end{gathered}
$$

In the case of no mixing, i.e., when $\cos \alpha=0$, then $v_{\phi}=v_{H}$, and the problem turns into studying the electroweak symmetry breaking in the scale invariant standard model, which has been investigated in $[18,19]$.

\section{CRITICAL TEMPERATURE AND WASHOUT CRITERION}

The strong first-order electroweak phase transition is one of the three Sakharov conditions [20] for the baryogenesis. For the $C P$ violation in the minimal scale invariant extensions of the SM, see [15]. The phase transition takes place at the critical temperature $T_{c}$ at which the free energy (effective potential) has two degenerate minima at $T=T_{c}$. In this section, we follow [21] to calculate analytically the washout criterion, i.e., $v\left(T_{c}\right) / T_{c}>1$, which guarantees the strong first-order phase transition.

The minimization condition on the thermal effective potential in Eq. (7), with the derivative being along the radial field,

$$
\left.\frac{\partial}{\partial \phi} V_{\mathrm{eff}}\right|_{v_{\phi}(T)}=0
$$

leads to a set of $T$-dependent equations for the vacuum expectation value,

$$
\begin{gathered}
v_{\mathrm{sym}}(T)=0, \\
v_{\mathrm{brk}}^{2}(T) \log \frac{v_{\mathrm{brk}}^{2}(T)}{v_{\phi}^{2}}=-\frac{C}{B} T^{2},
\end{gathered}
$$

\footnotetext{
${ }^{2}$ Note that we are considering a one-step phase transition along the flat direction $\phi$. In general, if more scalars in the model take a nonzero VEV, a two-step phase transition may be required. See [17], for instance.
}

where $v_{\text {sym }}$ is the VEV of the radial field in the symmetric phase and $v_{\text {brk }}$ is the VEV in the broken phase. In the SM, the $v_{\text {brk }}$ is the temperature-dependent vacuum expectation value of the Higgs doublet, but in our case $v_{\text {brk }}$ is the VEV along the flat direction, i.e., the VEV of the mixing of the Higgs doublet and the scalon. Equation (12) has no analytic solution for $v_{\text {brk }}$. Nevertheless, the solution can be expressed in terms of the Lambert W function, which is defined as

$$
z=w e^{w} \Leftrightarrow w=W(z),
$$

where $z$ and $w$ in general are complex numbers. In terms of the Lambert $W$ function, Eq. (12) is written as

$$
v_{\mathrm{brk}}^{2}=\frac{-C T^{2} / B}{W\left(-\frac{C}{B v_{\phi}^{2}} T^{2}\right)} .
$$

At the critical temperature $T_{c}$, the effective potential in Eq. (7) must be vanishing at the minimum $v_{\text {brk }}$ as it is vanishing also at the minimum $v_{\text {sym }}=0$ of the symmetric phase. Multiplying Eq. (12) by $v_{\phi}^{2}$ and substituting its righthand side in $V_{\text {eff }}\left(v_{\text {brk }}\right)=0$ from Eq. (7), we arrive at $v_{\mathrm{brk}}^{2}\left(T_{c}\right)=(2 C / B) T_{c}^{2}$. Therefore, the condition for the electroweak phase transition to be strongly first order (the washout criterion) becomes

$$
\frac{v_{\mathrm{brk}}\left(T_{c}\right)}{T_{c}}=\sqrt{\frac{2 C}{B}}>1 .
$$

Finally, substituting $v_{\text {brk }}$ from Eq. (15) into Eq. (7), expanding the effective potential, and setting that to zero, we obtain

$$
T_{c}^{2} \simeq(\sqrt{11}-3) \frac{B}{C} v_{\phi}^{2}
$$

Before going further with more constraints on the parameters, regarding the values of $B$ and $C$ in Eqs. (8) and (9), it is clear that the ratio $v_{c} / T_{c}$ can easily be large enough, leading to a very strong first-order phase transition.

\section{STABILITY CONDITIONS}

The stability conditions impose already strong constraints on the parameters of the model. The first derivative of the tree-level potential in Eq. (1) must vanish at the VEVs,

$$
\left.\frac{\partial V}{\partial h}\right|_{\langle h\rangle}=\left.\frac{\partial V}{\partial s}\right|_{\langle s\rangle}=0,
$$

which in turn leads to

$$
\lambda_{h} v_{h}^{2}=-\lambda_{h s} v_{s}^{2}, \quad \lambda_{s} v_{s}^{2}=-\lambda_{h s} v_{h}^{2} .
$$

The positivity of the second derivatives of the potential in Eq. (1) gives rise to 


$$
\lambda_{h s}<0, \quad \lambda_{s s^{\prime}}>0 .
$$

From Eqs. (19) and (4), we get $\lambda_{h}>0$. Now the radiative correction to the scalon mass in Eq. (5) is positive if $m_{s^{\prime}}>316.5 \mathrm{GeV}$. Using Eq. (4) for $m_{s^{\prime}}$, one arrives at $\lambda_{s s^{\prime}}>-1.65 \lambda_{h s} / \lambda_{h}$. Still we can make use of the Higgs mass relation in Eq. (4) to constrain more the Higgs coupling: $\lambda_{h}=\lambda_{h s}+0.128$. As $\lambda_{h}>0$ and $\lambda_{h s}<0$, then $-0.128<\lambda_{h s}<0$.

\section{DARK MATTER}

The scalar $s^{\prime}$ taking a zero expectation value is stable and can play the role of the thermal dark matter candidate within the freeze-out scenario. In this section we add the relic density condition to the washout criterion obtained in the previous section and probe the space of the parameters. The independent parameters in the model are not many; $\lambda_{h s}$, $\lambda_{s^{\prime}}$, and $\lambda_{s s^{\prime}}$, among which only the parameter $\lambda_{h s}$ takes part in the relic density computation. The dark matter sector interacts with the visible sector via the scalar mediator $s$, which has become massive through the radiative correction, and its mass is given by Eq. (5). In fact, the scalar mediator $s$ mixes with the Higgs field in the SM and the mixing angle is that of the flat direction in Eq. (3). The Higgs vacuum expectation value is known experimentally; $v_{h}=246 \mathrm{GeV}$ and the VEV of the scalar $s$ is determined by other known parameters of the theory as seen from Eq. (18).

The thermal evolution of the dark matter number density $n_{s^{\prime}}$ in the early Universe is given by the Boltzmann equation,

$$
\frac{d n_{s^{\prime}}}{d t}+3 H n_{s^{\prime}}=-\left\langle\sigma_{\mathrm{ann}} v_{\mathrm{rel}}\right\rangle\left[n_{s^{\prime}}^{2}-\left(n_{s^{\prime}}^{\mathrm{EQ}}\right)^{2}\right],
$$

where $H$ is the Hubble expansion rate, $v_{\text {rel }}$ stands for the dark matter relative velocity, and the $\sigma_{\mathrm{ann}}$ is the dark matter annihilation cross section. We compute the relic abundance using the MicrOMEGAs4 . 3 package [22] that numerically solves the Boltzmann differential equation. We recall that the potential we use to compute the relic density is the potential in Eq. (1) after the electroweak symmetry breaking, which is given by

$$
\begin{aligned}
V\left(h, s, s^{\prime}\right)= & \frac{1}{2} m_{h}^{2} h^{2}+\frac{1}{2} m_{s^{\prime}}^{2} s^{2}+\left(\lambda_{h}+\lambda_{h s}\right) \sqrt{1-\frac{\lambda_{h s}}{\lambda_{h}}} v_{h} h^{3}+\frac{1}{4} \frac{\left(\lambda_{h}+\lambda_{h s}\right)^{2}}{\lambda_{h}} h^{4} \\
& +\left(\lambda_{h}+\lambda_{h s}\right) \sqrt{-\frac{\lambda_{h s}}{\lambda_{h}} h^{3} s+2 \sqrt{-\lambda_{h s}\left(\lambda_{h}-\lambda_{h s}\right)} v_{h} h^{2} s} \\
& -\lambda_{h s} h^{2} s^{2}+\frac{\lambda_{h} \lambda_{s s^{\prime}} v_{h}}{\sqrt{-\lambda_{h s}\left(\lambda_{h}-\lambda_{h s}\right)}} s s^{\prime 2}-\frac{\lambda_{s s^{\prime}} \lambda_{h} v_{h}}{\sqrt{\lambda_{h}\left(\lambda_{h}-\lambda_{h s}\right)}} h s^{2}+\frac{\sqrt{-\lambda_{h s} \lambda_{h}} \lambda_{s s^{\prime}}}{\lambda_{h}-\lambda_{h s}} s h s^{\prime 2} \\
& +\frac{1}{2} \frac{\lambda_{h} \lambda_{s s^{\prime}}}{\lambda_{h}-\lambda_{h s}} s^{2} s^{\prime 2}-\frac{1}{2} \frac{\lambda_{s s^{\prime}} \lambda_{h s}}{\lambda_{h}-\lambda_{h s}} h^{2} s^{\prime 2}+\frac{1}{4} \lambda_{s^{\prime}} s^{\prime 4} .
\end{aligned}
$$

Note that the Higgs scalar field has a mass term now in Eq. (21). The phase transition (going from the symmetric phase with $v_{h}=0$ to the broken phase with $v_{h} \neq 0$ ) is followed by the scale symmetry breaking through the radiative correction to the scalon mass. We constrain the model by the observed dark matter relic abundance from the WMAP/Planck $[23,24]$ to be $\Omega_{\mathrm{DM}} h^{2} \sim 0.11$. In Sec. IV the mass of the dark matter already had a lower bound due to the positivity of the scalon mass: $m_{s}^{\prime} \equiv m_{\mathrm{DM}}>316.5 \mathrm{GeV}$. In Fig. 1 the dark matter mass is plotted against the only independent coupling, i.e., $\lambda_{h s}$. As seen from this figure, the viable range of the coupling shrinks to $-0.007 \lesssim \lambda_{h s} \lesssim 0$. The scalon could be searched for at the Large Hadron Collider (LHC) or future colliders via the exotic Higgs decays $h \rightarrow s s$ and $h \rightarrow s s s$. It has been pointed out in Ref. [25] that even couplings as small as $\mathcal{O}\left(10^{-2}\right)$ yield $\operatorname{Br}(h \rightarrow \mathrm{BSM}) \sim 10 \%$. So even very small Higgs-scalon coupling can in principle lead to a signature at the LHC. The DM mass, however, sits almost within the same limit we

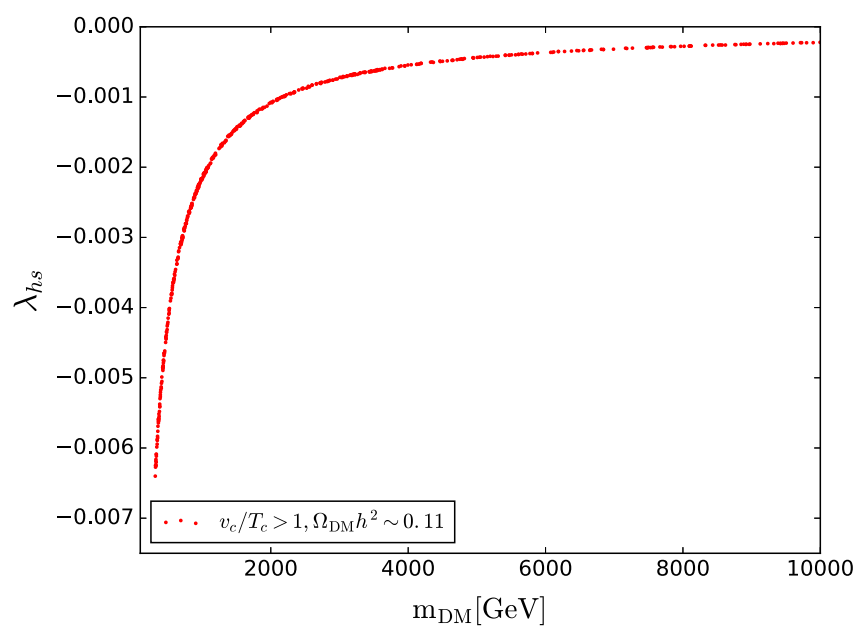

FIG. 1. The plot compares the dark matter mass against the coupling $\lambda_{h s}$ with the washout criterion satisfied and $\Omega_{\mathrm{DM}} h^{2} \sim 0.11$. 


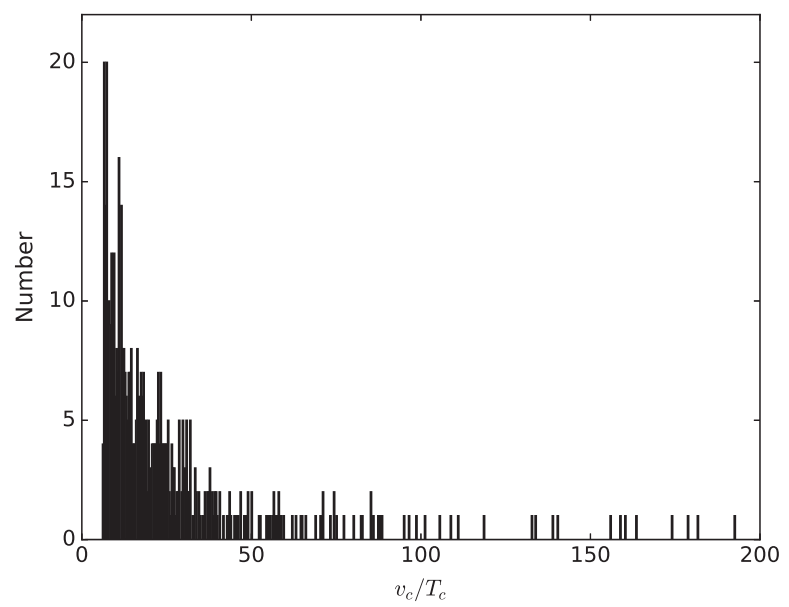

FIG. 2. A histogram for the ratio $v_{c} / T_{c}$ with the correct relic density $\Omega h^{2} \sim 0.11$. It is shown that $v_{c} / T_{c} \gtrsim 4$, which guarantees a very strong first-order phase transition.

obtained in Sec. IV, i.e., $m_{\mathrm{DM}}>318.3 \mathrm{GeV}$. In Fig. 2 we have also demonstrated a histogram of the values $v_{c} / T_{c}$ which are bounded by the correct relic density. It is observed that $v_{c} / T_{c}>3.8$; in fact, it takes much larger values which guarantees a very strong first-order electroweak phase transition.

\section{DIRECT DETECTION CONSTRAINT}

There are experiments that have been set up with the goal of detecting the elusive dark matter directly. Among these, the XENON1t experiment located at Gran Sasso in Italy is the most recent and the more accurate one [26]. Although the XENON1t experiment and no other experiments such as LUX (see [27] for the recent results) have not detected the dark matter, they have put a very stringent constraint on the elastic scattering cross section of the dark matter off the nucleus. We examine the current model by data from the direct detection experiments.

The DM-nucleus cross section can be described simply by the following effective potential,

$$
\mathcal{L}_{\text {eff }}=\alpha_{q} s^{\prime} s^{\prime} \bar{q} q
$$

where $q$ stands for the quark in the nucleon and $s^{\prime}$ is the dark matter field. The coupling $\alpha_{q}$ is given by

$$
\alpha_{q}=m_{q} \frac{2 \lambda_{h} \lambda_{s s^{\prime}}}{\lambda_{h}-\lambda_{h s}}\left(\frac{1}{m_{h}^{2}}-\frac{1}{m_{s}^{2}}\right)
$$

The DM-nucleus scattering is obtained from a treelevel Feynman diagram leading to the following spinindependent elastic scattering cross section:

$$
\sigma_{\mathrm{SI}}^{\mathrm{N}}=\frac{\alpha_{N}^{2} \mu_{N}^{2}}{\pi m_{\mathrm{DM}}^{2}}
$$

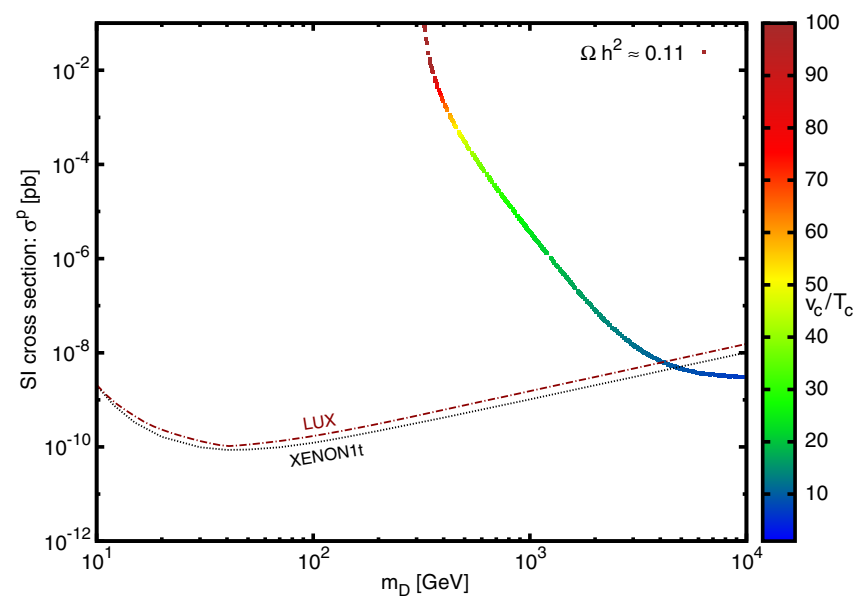

FIG. 3. The viable range of the DM mass is $m_{\mathrm{DM}} \gtrsim 4.5 \mathrm{TeV}$ after imposing the XENON1t/LUX direct detection experiments on the DM-nucleus elastic scattering cross section, the DM relic density constraint, and the first-order phase transition condition.

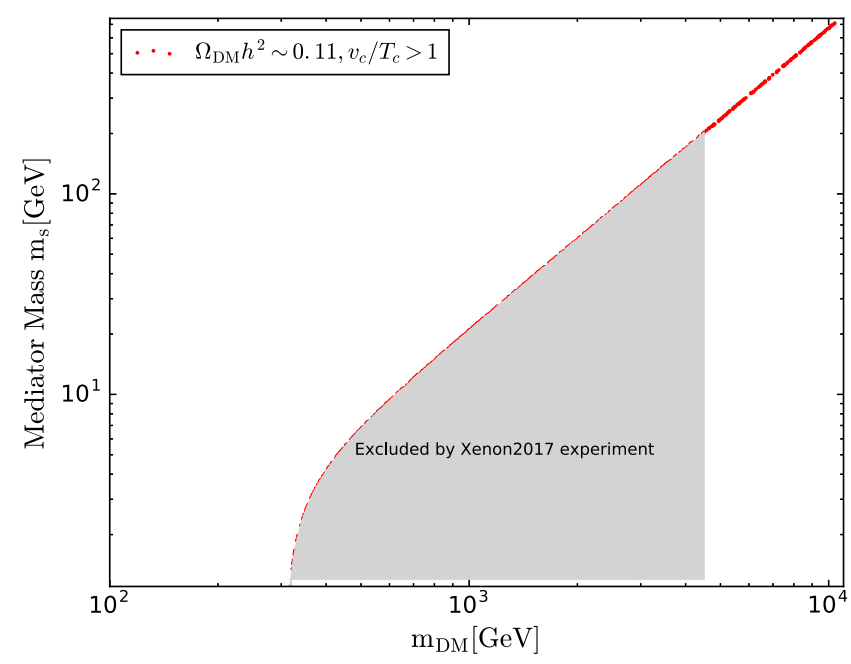

FIG. 4. The plot shows the viable range of the scalon mass being $m_{s} \gtrsim 200 \mathrm{GeV}$.

where $\mu_{N}$ is the reduced mass for the DM-nucleus system and $\alpha_{N}$ denotes a coefficient that depends on the nucleon form factors. For more details on $\alpha_{N}$, see [28] and the references therein.

For the viable parameter space that we obtained in Sec. V, we have computed the elastic scattering cross section in Eq. (24) using the MicrOMEGAs4 . 3 package. The result is shown in Fig. 3. According to Fig. 3, only the dark matter mass $m_{\mathrm{DM}} \gtrsim 4.5 \mathrm{TeV}$ survives the XENON1t/ LUX cross section limits, ${ }^{3}$ while respecting both the relic density constraint and the washout criterion. It is interesting also to determine the allowed masses of the scalon $s$, which is the mediator connecting the DM sector to the SM. As

\footnotetext{
${ }^{3}$ Thanks to Christopher Tunnell for providing me with the XENON1t data in Fig. 4 of [26].
} 
seen in Fig. 4, the scalon mass $m_{s}$ takes only values above $200 \mathrm{GeV}$.

\section{CONCLUSION}

In this paper we have studied the minimal extension of the scale invariant standard model with two extra scalars, $s$ and $s^{\prime}$, in addition to the Higgs particle. Two scalars are the minimum number of scalars that we should add to the scale invariant standard model to give a mass of $125 \mathrm{GeV}$ to the Higgs and correct masses for other particles in the SM. The classically massive scalar $s^{\prime}$ is interpreted as a dark matter candidate and the classically massless scalar $s$, called the scalon, plays the role of the DM-SM mediator. We showed that this model supports a very strong first-order electroweak phase transition even if we constrain the model with the observed DM relic density by WMAP/Planck. Imposing the limits from the direct detection experiments such as XENON1t/LUX on the elastic scattering cross section of the DM-nucleus still allows the dark matter mass $m_{\mathrm{DM}} \gtrsim 4.5 \mathrm{TeV}$ and the scalon mass $m_{s} \gtrsim 200 \mathrm{GeV}$.

\section{ACKNOWLEDGMENTS}

I am very grateful to Karim Ghorbani for many fruitful discussions. I would also like to thank Ville Vaskonen for useful email correspondence.
[1] G. Aad et al. (ATLAS Collaboration), Observation of a new particle in the search for the Standard Model Higgs boson with the ATLAS detector at the LHC, Phys. Lett. B 716, 1 (2012).

[2] S. Chatrchyan et al. (CMS Collaboration), Observation of a new boson at a mass of $125 \mathrm{GeV}$ with the CMS experiment at the LHC, Phys. Lett. B 716, 30 (2012).

[3] F. Englert and R. Brout, Broken Symmetry and the Mass of Gauge Vector Mesons, Phys. Rev. Lett. 13, 321 (1964).

[4] P. W. Higgs, Broken Symmetries and the Masses of Gauge Bosons, Phys. Rev. Lett. 13, 508 (1964).

[5] K. A. Meissner and H. Nicolai, Effective action, conformal anomaly and the issue of quadratic divergences, Phys. Lett. B 660, 260 (2008).

[6] W. A. Bardeen, On naturalness in the standard model, in Ontake Summer Institute on Particle Physics Ontake Mountain, Japan, 1995 (1995), http://lss.fnal.gov/cgi-bin/find_ paper.pl?conf-95-391.

[7] S. R. Coleman and E. J. Weinberg, Radiative corrections as the origin of spontaneous symmetry breaking, Phys. Rev. D 7, 1888 (1973).

[8] E. Gildener and S. Weinberg, Symmetry breaking and scalar bosons, Phys. Rev. D 13, 3333 (1976).

[9] A. Farzinnia, H.-J. He, and J. Ren, Natural electroweak symmetry breaking from scale invariant higgs mechanism, Phys. Lett. B 727, 141 (2013).

[10] F. Sannino and J. Virkajarvi, First order electroweak phase transition from (Non)conformal extensions of the standard model, Phys. Rev. D 92, 045015 (2015).

[11] E. Gabrielli, M. Heikinheimo, K. Kannike, A. Racioppi, M. Raidal, and C. Spethmann, Towards completing the standard model: vacuum stability, EWSB and dark matter, Phys. Rev. D 89, 015017 (2014).

[12] K. Endo and K. Ishiwata, Direct detection of singlet dark matter in classically scale-invariant standard model, Phys. Lett. B 749, 583 (2015).

[13] K. Hashino, M. Kakizaki, S. Kanemura, and T. Matsui, Synergy between measurements of gravitational waves and the triple-Higgs coupling in probing the first-order electroweak phase transition, Phys. Rev. D 94, 015005 (2016).

[14] K. Ghorbani and H. Ghorbani, Scalar dark matter in scale invariant standard model, J. High Energy Phys. 04 (2016) 024.

[15] L. Alexander-Nunneley and A. Pilaftsis, The minimal scale invariant extension of the standard model, J. High Energy Phys. 09 (2010) 021.

[16] L. Marzola, A. Racioppi, and V. Vaskonen, Phase transition and gravitational wave phenomenology of scalar conformal extensions of the Standard Model, Eur. Phys. J. C 77, 484 (2017).

[17] W. Chao, H.-K. Guo, and J. Shu, Gravitational wave signals of electroweak phase transition triggered by dark matter, J. Cosmol. Astropart. Phys. 09 (2017) 009.

[18] K. Endo and Y. Sumino, A scale-invariant higgs sector and structure of the vacuum, J. High Energy Phys. 05 (2015) 030.

[19] K. Hashino, S. Kanemura, and Y. Orikasa, Discriminative phenomenological features of scale invariant models for electroweak symmetry breaking, Phys. Lett. B 752, 217 (2016).

[20] A. D. Sakharov, Violation of CP Invariance, c Asymmetry, and Baryon Asymmetry of the Universe, Usp. Fiz. Nauk 161, 61 (1991) [Pis'ma Zh. Eksp. Teor. Fiz. 5, 32 (1967)].

[21] P. H. Ghorbani, Electroweak baryogenesis and dark matter via a pseudoscalar vs. scalar, J. High Energy Phys. 08 (2017) 058.

[22] G. Belanger, F. Boudjema, A. Pukhov, and A. Semenov, micrOMEGAs4.1: two dark matter candidates, Comput. Phys. Commun. 192, 322 (2015).

[23] G. Hinshaw et al. (WMAP Collaboration), Nine-year wilkinson microwave anisotropy probe (wmap) observations: Cosmological parameter results, Astrophys. J. Suppl. Ser. 208, 19 (2013).

[24] R. Adam et al. (Planck Collaboration), Planck 2015 results. I. Overview of products and scientific results, Astron. Astrophys. 594, A1 (2016). 
[25] D. Curtin et al., Exotic decays of the $125 \mathrm{GeV}$ Higgs boson, Phys. Rev. D 90, 075004 (2014).

[26] E. Aprile et al. (XENON Collaboration), First Dark Matter Search Results from the XENON1T Experiment, Phys. Rev. Lett. 119, 181301 (2017).
[27] D. S. Akerib et al. (LUX Collaboration), Results from a Search for Dark Matter in the Complete LUX Exposure, Phys. Rev. Lett. 118, 021303 (2017).

[28] K. Ghorbani, Fermionic dark matter with pseudo-scalar Yukawa interaction, J. Cosmol. Astropart. Phys. 01 (2015) 015. 